\title{
Simple and Short-term Inspiration Training Accelerates Recovery from Residual Pulmonary Hypertension after Mitral Valve Surgery: A Randomized Control Trial
}

\author{
Basuni Radi 1,2, Bima Suryaatmaja ${ }^{3}$, \\ Ario S Koencoro' ${ }^{1,2}$, Amiliana M Soesanto ${ }^{1,2}$
}

\footnotetext{
' National Cardiovascular Center Harapan Kita, Jakarta, Indonesia 2 Department of Cardiology and Vascular Medicine, Faculty of Medicine, Universitas Indonesia, Jakarta, Indonesia

${ }^{3}$ Bendan Hospital, Pekalongan City, Indonesia
}

\section{Correspondence:}

Basuni Radi,

National Cardiovascular Center Harapan Kita, Jakarta, Indonesia, II 420

Email: basuni_radi@hotmail.com / basuni.radi@pjnhk.go.id

\begin{abstract}
Background: Residual pulmonary hypertension after mitral valve surgery predicts a worse prognosis. This study aimed to explore the effect of inspiratory muscle training on functional capacity and pulmonary artery pressure in patients with residual pulmonary hypertension after mitral valve surgery.

Methods: Forty-three patients with residual pulmonary hypertension soon after mitral valve repair or replacement surgery were consecutively enrolled in this randomised controlled trial. They were randomly allocated to either an intervention group $(n=22)$ or a control group $(n=2 I)$. Both groups participated in usual $10-12$ sessions of supervised aerobic exercise training, with low to moderate intensity. The subjects in the intervention group participated in additional inspiratory muscle training using an incentive spirometer with a series of $10-12$ inspiration repetitions of $50 \%$ of maximum inspiratory volume in each aerobic session. Echocardiography and the 6-minute walking test were performed before and after the programme.

Results: The patients were $70 \%$ female, mean age $43.5 \pm 13$ years. Both groups had similar baseline characteristics. The systolic pulmonary artery pressure declined from $5 \mathrm{I}(43-68) \mathrm{mmHg}$ to $35(29-39) \mathrm{mmHg}, \mathrm{p}<0.00 \mathrm{I}$ in intervention group, and from $46(43-55) \mathrm{mmHg}$ to $43(40-5 \mathrm{I}) \mathrm{mmHg}, \mathrm{p}<0.0 \mathrm{I}$ in control group. The reduction was higher in the intervention group than in the control group, $16(\mathrm{I} 2-30)$ vs $3.5(2-4) \mathrm{mmHg}$ respectively, $\mathrm{p}<0.00 \mathrm{I}$, and in patients with higher baseline systolic pulmonary artery pressure. The 6-minute walk test distance increased from 308 (242-353) meters to 407 (377-433) meter, $\mathrm{p}<0.0 \mathrm{I}$ in intervention group, and from 353 (334-38I) meter to 39I (376-429) meter, $\mathrm{p}<0.0 \mathrm{I}$ in control group. The improvement was higher in the intervention group than in control group, I0I (93-I3I) vs 48 (35-53) meter respectively, $\mathrm{p}<0.00 \mathrm{I}$.

Conclusion: Adding inspiratory muscle training to a usual exercise programme resulted in faster recovery from residual pulmonary hypertension and higher increase in functional capacity.
\end{abstract}

(Indonesian J Cardiol. 202I;42: I5-23)

Keywords: inspiratory muscle training, pulmonary hypertension; mitral valve surgery; exercise. 


\section{Introdution}

$\mathrm{P}$ ulmonary hypertension $(\mathrm{PH})$ is a common complication of mitral valve diseases. In general, pulmonary artery pressure (PAP) declines after mitral valve surgery or non-surgical intervention. ${ }^{1}$ However, in some cases, reduction in PAP is delayed and $\mathrm{PH}$ persists until 6-12 months after the interventions, ${ }^{2}$ and there is an increase in the mortality and morbidity rate in patients with residual $\mathrm{PH}$ after mitral valve surgery. ${ }^{3}$

Pathological and functional changes in the pulmonary vasculature and right heart are initiated by an increase in the left ventricle or left atrial filling pressure and followed by elevated pulmonary venous pressure. ${ }^{4}$ These changes may also continue to an irreversible remodelling of the alveolar-capillary membrane, which is characterised by excessive deposition of type IV collagen. ${ }^{5}$ Additional risk of pulmonary dysfunction is also associated with open heart surgery procedures such as phrenic nerve dysfunction, pulmonary endothelial dysfunction, atelectasis, or restrictive ventilatory pattern which is more frequent in patients who have undergone valve surgeries. These conditions may slow recovery from $\mathrm{PH}$ after valvular surgery. ${ }^{6}$

Aerobic exercise with or without respiratory training improves the physical capacity and quality of life in patients with various forms of $\mathrm{PH} .{ }^{7,8}$, However, the evidence of respiratory training's effects on recovery from residual PH after mitral valve surgery is sparse. This study aimed to explore the effect of additional inspiratory muscle training on usual aerobic exercise training in a cardiac rehabilitation programme (CRP) for recovery from $\mathrm{PH}$ and the functional capacity of patients with residual $\mathrm{PH}$ after mitral valve surgery.

\section{Methods}

\section{Study Design}

This study was a randomised controlled trial that recruited patients after mitral valve repair or replacement surgery who were registered to participate in phase II exercise-based CRP in the National Cardiovascular Centre Harapan Kita Jakarta (NCVCHK), between May 2015 to October 2015. This research was approved by the Board of Research Ethics of the NCVCHK. The minimum sample size estimation was 19 per group to provide $80 \%$ power at the $5 \%$ (two-sided) level of significant to a difference in pulmonary artery pressure change.

A set of even or odd random numbers in a sealed envelope for each number was ordered to randomise the patients. Patients who met the recruitment criteria would consecutively be allocated to the intervention group if they received even numbers or to the control group for odd numbers.

\section{Patients}

For this study, the patients were consecutively recruited if they were older than 18 years, with systolic $\mathrm{PAP}>39 \mathrm{mmHg}$ in pre-participation echocardiography, left ventricle ejection fraction $>40 \%$, able to participate in CRP, and consented to participate in this study after receiving appropriate information. They were excluded if they had any contraindications to exercise and respiratory training (such as orthopaedic or communication problems), significant residual mitral stenosis or regurgitation, severe infection, or congenital heart disease. The eligible patients were then randomly allocated to either an inspiratory muscle training group (IMT) or a control group (CG).

\section{Procedures}

In the NCVCHK, early phase II CRP with aerobic exercise is a standard service for all patients after cardiac surgeries unless contraindicated. Patients are commonly registered within the first week after hospital discharge after seeing a cardiologist and cardiac surgeon in the outpatient clinic. The CRP consists of education and counselling sessions and aerobic exercise training sessions. The hospital-based CRP is covered by national health insurance for only 12 sessions, so most of the patients participate in the CRP for up to 12 sessions only and continue the exercise programme in a homebased CRP.

The aerobic exercise session consisted of approximately 10 minutes of warming up and stretching, 10 minutes of ergo-cycling with a workload of approximately 10-25 Watts that could be adjusted by the supervisor based on the patients' perceived exertion, and 20-30 minutes of corridor or treadmill walking in a 
gymnasium at their comfortable speed with Borg Scale 11-13 or with an increasing heart rate of 10-20 beats per minute above resting. The session ended with 5 minutes of cooling down/relaxation. The walking distance was usually increased by 100-200 meters at each following session. There were 5 CRP sessions per week that were supervised by trained nurses and physiotherapists.

All of the patients participated in this standard aerobic exercise, but those in the IMT group underwent additional inspiratory muscle training after each aerobic exercise session. The inspiratory muscle training was performed using RCI Voldyne 5000 equipped only with an inspiratory volume indicator. The spirometer selection was based on its availability.

To identify the maximum inspiratory volume (MIV), all of the patients were tested using similar equipment. They were instructed to take five maximum inspirations, and the MIV was calculated as the average of the five inspirations.

The inspiratory muscle training consisted of three series of 12 inspiration repetitions of $50 \%$ of the average MIV in a seated position. The volume indicator was set to a specific volume of $50 \%$ of the MIV. For one inspiratory training repetition, the patients were instructed to place the spirometer mouthpiece tightly after maximum exhalation, then take a deep, slow inhalation until the main piston reached the marked goal and the other smaller piston was within the "Better" and "Best" range sign, hold their breath for 3-5 seconds, and then exhale. The 12 repetitions were performed completely, except when the patients felt discomfort such as fatigue, dizziness, or coughing. The training then resumed when the patients felt better and were able to continue.

The inspiratory muscle training usually took 15 20 minutes and was supervised by physiotherapists. The inspiratory muscle training schedule followed each aerobic exercise session, which was carried out for 5 sessions per week so all of the sessions could be finished in 2.5 weeks.

The CG patients received the usual CRP care. They participated in 10-12 sessions of supervised aerobic exercise in early and short-term phase II exercise-based CRP, with additional sham inspiratory muscle training that amounted to only $15 \%$ of the MIV after each aerobic exercise session with a procedure similar to that of the IMT patients.
The patients' medications were supplied for 1 month following discharge from the outpatient clinic.

\section{Measurement}

The echocardiography examinations, MIV test, and 6-minute walking test (6-MWT) were performed before and after the programme.

The echocardiography examinations were carried out by a senior sonographer and supervised by cardiologists who were blinded to the patient groups using a GE Vivid 7 Dimension $3.0 \mathrm{MHz}$ transducer. The left ventricle systolic function was evaluated by calculating the left ventricle ejection fraction (LVEF) using the Teichholz method (M-mode) or Simpson's method (2D echocardiography). The right ventricle function was evaluated by measuring the tricuspid annular plane systolic excursion (TAPSE) in the M-mode. The assessment of $\mathrm{PH}$ including systolic PAP, mean PAP, and tricuspid valve gradient and grading were based on the 2015 ESC/ERS Guidelines for the Diagnosis and Treatment of Pulmonary Hypertension.9

Systolic PA pressure was calculated by a formula: Systolic PAP $=$ TR V max + e RAP. Tricuspid regurgitation maximum velocity (TR $\mathrm{V} \max$ ) was a measurement of maximum velocity of tricuspid regurgitation using continuous Doppler echocardiography. eRAP was the measurement of inferior vena cava diameter at inspiration, and its collapsibility by sniffing.

Echocardiography measurement was performed one day prior to participation in the cardiac rehabilitation program.

The 6-MWTs were carried out in a 30-meter-long gymnasium corridor tract. The patients were instructed to walk as far as possible within 6 minutes. Standard verbal encouragement was provided, and the remaining time was announced each minute, except during the last minute, when it was announced every 15 seconds. The patients were allowed to stop or rest during the test when they needed and resumed walking when possible. Their heart rate and rhythm were monitored using ECG telemetry. The patients underwent similar tests before hospital discharge for familiarisation and programme orientation.

\section{Statistical Analysis}

The baseline characteristics' numerical data are presented as the mean+standard deviation (SD) and 
as the median and 25th and 75th percentile when the data distribution was not normal, as for the BMI data. The categorical data are presented as the frequency and percentage.

A comparison of the numerical data between the groups was performed using Student's independent t-test and the Mann-Whitney test when the data distribution was not normal. The categorical data were compared using the Chi-squared test or Fischer's test and the paired t-test to compare numerical pre- and post-data within group or the Wilcoxon signed-rank test when the data distribution was not normal. $\mathrm{P}<0.05$ was considered statistically significant.

A multivariate analysis with a linear regression model was performed to analyse which determinants independently affected the reduction in systolic PAP and the improvement in the 6-MWT distance. This analysis included inspiratory muscle training, gender, age, pulmonary vasodilator agent use, atrial fibrillation, baseline systolic PAP, and baseline 6-MWT distance.

\section{Results}

There were 48 patients who met the recruitment criteria presented consecutively. but 5 of them could not participate in this study due to personal reasons, so that a total of 43 patients were recruited and randomly allocated to the IMT $(n=21)$ and control groups $(n=22)$. They were $70 \%$ female, their age ranged from 18 to 67 years, a body mass index of $21.4+5.0 \mathrm{~kg} / \mathrm{m} 2$, and LVEF of $57.5+11.2 \%$. The aetiology of mitral valve pathology was rheumatic heart disease in all 18 patients with mitral valve stenosis and in $13(52 \%)$ of patients with mitral regurgitation. Meanwhile, the rest of the patients with mitral regurgitation was caused by degenerative disease which presented as mitral valve prolapse.

None of the patients had a history of asthma or chronic obstructive pulmonary disease and thromboembolism, but 5 (12\%) had hypertension, 5 $(12 \%)$ had diabetes, and $3(7 \%)$ were smokers. Thirtytwo of the patients underwent mitral valve replacement surgery, and 11 patients underwent mitral valve repair surgery. They were treated with diuretics $(86 \%)$, anticoagulants $(100 \%)$, angiotensin-converting enzyme inhibitors (86\%), pulmonary vasodilators $(23 \%)$, and beta-blocking agents (62\%) at the beginning of this study. All of the medications were continued during the study period without significant changes in both groups except anti-coagulant adjustment doses.

The systolic PAP of all of the patients declined significantly from pre-surgery $(64.1+19.6 \mathrm{mmHg})$ to post-surgery/pre-study $(51.5+11.7 \mathrm{mmHg})$ and to poststudy $(40.1+10.1 \mathrm{mmHg}), \mathrm{P}<0.001$.

There was improvement in the right ventricle (RV) function as demonstrated by an increase in TAPSE from $1.1+0.25 \mathrm{~cm}$ pre-study to $1.3+0.28 \mathrm{~cm}$ post-study, $\mathrm{P}<0.01$, and improvement in the exercise capacity as demonstrated by increased 6-MWT distance from $326.9+58.3$ meters pre-study to $402.0+58.3$ meters post-study, $\mathrm{P}<0.01$.

The baseline characteristics of the two groups were comparable Table 1, except the 6-MWT distance, which was significantly higher in the CG patients. Because of this difference, the 6-MWT distance was included in the multiple linear regression analysis (Table 3 and Table 4) to clarify whether this variable influenced the improvement in the 6-MWT distance and systolic PAP.

The comparison of the effects of the specific intervention on each group is presented in Table 2. Both groups demonstrated significant improvements in every parameter, but the changes were significantly higher or better in the IMT group. The IMT and CG patients had comparable systolic PAP before surgery $(64.6+16.5$ $\mathrm{mmHg}$ vs $63.6+22.5 \mathrm{mmHg}, \mathrm{P}=0.872$ ) and pre-study $(54.8+13.8 \mathrm{mmHg}$ vs $48.3+8.4 \mathrm{mmHg}, \mathrm{P}=0.07)$, but significantly lower in the IMT group post-study $(35.0+9.9 \mathrm{mmHg}$ vs $45.0+7.8 \mathrm{mmHg}, \mathrm{P}=0.001)$. There was no change in the LVEF after intervention in the IMT group $(57.7+12.5$ to $57.4+11.9$ meters, $\mathrm{P}=0.897)$ and in the control group $(57.4+9.9$ to $59.8+9.3$ meters, $\mathrm{P}=0.161$ ).

Inspiratory muscle training combined with aerobic exercise training in the IMT group also resulted in a better reduction in the mean PAP, maximum velocity of tricuspid regurgitation (MVTR), and tricuspid valve gradient (TVG) than in the CG patients.

Improvement in TAPSE was also significantly better in the IMT group than in the CG patients. The baseline 6-MWT distance was significantly better in the CG patients; however, there was a greater increase with IMT, which resulted in a similar functional capacity in both groups at the end of the study. The MIV increased to a significantly higher level in the RT group and resulted in a higher MIV at the end of the study. 
Table 1. Baseline characteristics

\begin{tabular}{|c|c|c|}
\hline Variables & $\begin{array}{c}\text { IMT group } \\
\mathbf{n}=21\end{array}$ & $\begin{array}{c}\begin{array}{c}\text { Control group } \\
\mathrm{n}=\mathbf{2 2}\end{array} \\
\end{array}$ \\
\hline Female gender & $17(81 \%)$ & $13(59 \%)$ \\
\hline Age (years) & $45 \pm 13$ & $42 \pm 12$ \\
\hline Body mass index $(\mathrm{kg} / \mathrm{m} 2)$ & $20(17-24)$ & $20(18-25)$ \\
\hline Systolic blood pressure & $104 \pm 12$ & $108 \pm 15$ \\
\hline Diastolic blood pressure & $61 \pm 8$ & $65 \pm 12$ \\
\hline \multicolumn{3}{|l|}{ Mitral valve disease } \\
\hline - $\quad$ Mitral stenosis & $8(38 \%)$ & $10(45 \%)$ \\
\hline - $\quad$ Mitral regurgitation & $13(62 \%)$ & $12(55 \%)$ \\
\hline \multicolumn{3}{|l|}{ Surgery type } \\
\hline - MV replacement & $16(76 \%)$ & $16(72 \%)$ \\
\hline - $\quad$ MV repair & $5(24 \%)$ & $6(28 \%)$ \\
\hline \multicolumn{3}{|l|}{ Echocardiography } \\
\hline$-\quad \operatorname{LVEF}(\%)$ & $57.7 \pm 12.5$ & $57.4 \pm 9.9$ \\
\hline - $\quad$ LA diameter $(\mathrm{mm})$ & $46 \pm 8$ & $48 \pm 8$ \\
\hline - $\quad$ TAPSE $(\mathrm{cm})$ & $1.1 \pm 0.3$ & $1.1 \pm 0.2$ \\
\hline \multicolumn{3}{|l|}{ Rhythm } \\
\hline - Atrial fibrillation & $9(43 \%)$ & $13(59 \%)$ \\
\hline - $\quad$ Sinus rhythm & $12(57 \%)$ & $9(41 \%)$ \\
\hline \multicolumn{3}{|l|}{ Treatment } \\
\hline - $\quad$ ACE inhibitor & $18(85.7 \%)$ & $19(86.4 \%)$ \\
\hline - Beta-blockers & $13(62 \%)$ & $14(63 \%)$ \\
\hline - Anti-coagulant & $21(100 \%)$ & $22(100 \%)$ \\
\hline - Anti-platelet & $1(51 \%)$ & $1(51 \%)$ \\
\hline - Diuretic & $19(90 \%)$ & $18(89 \%)$ \\
\hline - Pulmonary vasodilator & $4(19 \%)$ & $6(27 \%)$ \\
\hline
\end{tabular}

Numerical data are presented as mean +standard deviation and as median (25th percentile and 75th percentile) when the data distribution is not normal. Categorical data are presented in frequency (percentage). RT: respiratory training group; ET: exercise training group; MV: mitral valve; LVEF: left ventricle ejection fraction; LA: left atrium; TAPSE: tricuspid annular plane systolic excursion; 6-MWT: 6-minute walking test; ACE: angiotensin-converting enzyme.

The multivariate linear regression analysis for all of the subjects (Table 3) and for the IMT patients only (Table 4) demonstrated that inspiratory muscle training independently contributed to the improvement in the systolic PAP and 6-MWT distance. The baseline systolic PAP was related to the level of improvement in systolic PAP after intervention, which demonstrated that the patients with worse conditions (higher baseline systolic PAP) obtained higher reduction in systolic PAP. The baseline 6-MWT distance was not related to the improvement in the 6-MWT distance or the systolic PAP reduction. Age tended to effect negatively to the improvement in the 6-MWT distance $(\mathrm{P}=0.099)$. There were 5 patients aged $>60$ years. However, compared to the younger patients, their baseline 6-MWT distance was comparable $(318+45$ vs $328+60.3$ meters, $\mathrm{P}=0,69)$, also the 6 -MWT distance improvement $(81.8+29.8$ vs $74.2+44$ meters, $\mathrm{P}=0.4$ )

\section{Discussion}

This randomised controlled study investigated the effects of aerobic exercise and additional inspiratory muscle training in patients with residual pulmonary hypertension after mitral valve surgery. The main results suggest that a standard cardiac rehabilitation programme with aerobic exercise (usual care) improves functional capacity, which was reflected by improvement in the 6-MWT distance and decreased the systolic PAP. The patients who received additional inspiratory muscle training obtained a better improvement in those parameters than the patients with usual care, although previously the systolic PAP had already declined significantly from $64.1+19.6 \mathrm{mmHg}$ before surgery to $51.5+11.7 \mathrm{mmHg}$ as an acute effect of surgery.

In this study, the mitral valve pathology was mitral stenosis $(58 \%)$ or mitral regurgitation $(42 \%)$, which was 
Indonesian Journal of Cardiology

Table 2. Baseline and post-intervention characteristics comparison

\begin{tabular}{|c|c|c|c|c|c|}
\hline Variables & $\begin{array}{c}\text { IMT group } \\
n=21\end{array}$ & $\mathbf{P}^{*}$ & $\begin{array}{c}\text { Control group } \\
\mathbf{n}=22\end{array}$ & $\mathbf{P}^{*}$ & $\mathbf{P}^{* *}$ \\
\hline SPAP pre, $\mathrm{mmHg}$ & $51(43-68)$ & $<0.01$ & $46(43-55)$ & $<0.01$ & 0.210 \\
\hline SPAP post, $\mathrm{mmHg}$ & $35(29-39)$ & & $43(40-51)$ & & 0.001 \\
\hline$\triangle$ SPAP, $\mathrm{mmHg}$ & $-16(-12,-30)$ & & $-3.5(-2,-4)$ & & $<0.001$ \\
\hline MPAP pre, $\mathrm{mmHg}$ & $40(30-47)$ & $<0.01$ & $35(30-40)$ & $<0.01$ & 0.910 \\
\hline MPAP post, $\mathrm{mmHg}$ & $25(18-30)$ & & $30(30-40)$ & & $<0.001$ \\
\hline$\triangle \mathrm{MPAP}(\mathrm{mmHg})^{\circ}$ & $-15(-10,-20)$ & & $0(0-5)$ & & $<0.001$ \\
\hline TRVmax pre, $\mathrm{m} / \mathrm{s}$ & $3(2.7-3.7)$ & $<0.01$ & $2.9(2.7-3.2)$ & $<0.01$ & 0.601 \\
\hline $\mathrm{TRV} \max$ post, $\mathrm{m} / \mathrm{s}$ & $2.5(2.2-2.7)$ & & $2.8(2.5-3.0)$ & & 0.003 \\
\hline$\Delta \mathrm{TRV} \max , \mathrm{m} / \mathrm{s}$ & $-0.6(-0.3,-1.1)$ & & $-0.2(-0.1,-0.3)$ & & $<0.001$ \\
\hline TVG pre, $\mathrm{mmHg}$ & $36(28-53)$ & $<0.01$ & $35(29-42)$ & $<0.01$ & 0.626 \\
\hline TVG post, $\mathrm{mmHg}$ & $25(20-28)$ & & $30(26-40)$ & & 0.004 \\
\hline$\Delta \mathrm{TVG}, \mathrm{mmHg}$ & $-13(-6,-27)$ & & $-4(-1.8,-7)$ & & $<0.001$ \\
\hline TAPSE pre, cm & $1.1 \pm 0.3$ & $<0.01$ & $1.1 \pm 0.2$ & 0.011 & 0.798 \\
\hline TAPSE post, $\mathrm{cm}$ & $1.4(1.2-1.7)$ & & $1.3(1.0-1.3)$ & & 0.092 \\
\hline$\triangle$ TAPSE, $\mathrm{cm}$ & $0.2(0-0.4)$ & & $0(0-0.2)$ & & 0.028 \\
\hline 6-MWT pre, m & $308(242-353)$ & $<0.01$ & $353(334-381)$ & $<0.01$ & 0.002 \\
\hline $6 \mathrm{MWT}$ post, $\mathrm{m}$ & $407(377-453)$ & & $391(376-429)$ & & 0.189 \\
\hline$\Delta 6 \mathrm{MWT}, \mathrm{m}$ & $101(93-131)$ & & $48(35-53)$ & & $<0.001$ \\
\hline MIV pre, mL & $750(750-1000)$ & $<0.01$ & $750(750-1000)$ & $<0.01$ & 0.657 \\
\hline MIV post, mL & $1500(1250-1500)$ & & $1125(1000-1286)$ & & 0.001 \\
\hline$\Delta \mathrm{MIV}, \mathrm{mL}$ & $500(500-700)$ & & $250(200-300)$ & & $<0.001$ \\
\hline
\end{tabular}

Numerical data are presented as mean (standard deviation) or median (25th percentile and 75th percentile). RT: respiratory training group; ET: exercise training group; pre: before intervention; post: after intervention; SPAP: systolic pulmonary artery pressure; MPAP: mean pulmonary artery pressure; TRVmax: tricuspid regurgitation maximum velocity; TVG: tricuspid valve gradient; TAPSE: tricuspid annular plane systolic excursion; 6-MWT: 6-minute walking test; MIV: maximum inspiratory volume; delta ( $\triangle)$ : change from pre-intervention to post-intervention. *P: value for comparison before and after within group. **P: value for comparison between groups. All P using the Wilcoxon signed-rank test.

Table 3. Multivariate analysis of the determinants of systolic pulmonary artery pressure and 6-MWT distance changes for all of the patients

\begin{tabular}{lcccc}
\hline & \multicolumn{2}{c}{ SPAP change } & & 6-MWT distance change \\
\hline \multicolumn{1}{c}{ Variables } & Standardised coefficient & $\mathbf{P}$ & Standardised coefficient & P \\
\hline Respiratory training & $\beta$ & $<0.01$ & 0.758 & $<0.001$ \\
Male gender & 0.630 & 0.982 & 0.068 & 0.516 \\
Age (year) & -0.002 & 0.299 & -0.170 & 0.099 \\
Pulmonary vasodilator & 0.091 & 0.768 & -0.164 & 0.134 \\
Atrial fibrillation & 0.028 & 0.792 & -0.022 & 0.841 \\
SPAP baseline & 0.025 & $<0.01$ & -0.032 & 0.784 \\
6-MWT baseline & 0.427 & 0.974 & -0.114 & 0.285 \\
\hline
\end{tabular}

SPAP: systolic pulmonary artery pressure; 6-MWT: six-minute walking test.

predominantly caused by rheumatic heart disease. In this type of illness, the duration of valvular abnormality is usually difficult to determine because of patient awareness and minimum symptoms at the beginning of the disease process or limited access to healthcare services. Abnormalities in respiratory function in patients with valvular heart diseases are frequently found, which are caused by an increase in pulmonary vascular resistance, fibrosis, and respiratory muscle wasting. ${ }^{6}$ Patients may recover within three months after surgery, although this is still below the predicted value.

Aerobic exercise is a standard procedure in cardiac rehabilitation programmes for patients after cardiac surgery and helps those with heart failure improve 
Indonesian Journal of Cardiology

Table 4. Multivariate analysis of the determinants of systolic pulmonary artery pressure and 6-MWT distance changes for the IMT patients

\begin{tabular}{lcccc}
\hline & \multicolumn{2}{c}{ SPAP change } & \multicolumn{2}{c}{ 6-MWT distance change } \\
\hline \multicolumn{1}{c}{ Variables } & Standardised coefficient & \multirow{2}{*}{$\mathbf{P}$} & Standardised coefficient & \multirow{2}{*}{$\mathbf{P}$} \\
\hline Male gender & 0.084 & & -0.004 & 0.985 \\
Age (year) & 0.284 & 0.643 & -1.769 & 0.099 \\
Pulmonary vasodilator & -0.033 & 0.138 & -1.713 & 0.109 \\
Atrial fibrillation & 0.094 & 0.891 & 0.273 & 0.322 \\
SPAP baseline & 0.806 & 0.684 & -0.317 & 0.238 \\
6-MWT baseline & 0.189 & 0.002 & -0.309 & 0.200 \\
\hline
\end{tabular}

SPAP: systolic pulmonary artery pressure; 6-MWT: six-minute walking test.

functional capacity. Aerobic exercise can also improve functional capacity in patients with idiopathic $\mathrm{PH},{ }^{10,8,7}$ so this training is recommended in the management of PH patients and those with chronic heart failure. ${ }^{?}$

The potential mechanisms on how exercise programmes improve functional capacity in patients with $\mathrm{PH}$ are similar to the mechanisms in patients with chronic heart failure who frequently have myopathy, decreased muscle perfusion, muscle fibre changes, and atrophy caused by cardiac conditions. ${ }^{11}$ Aerobic exercise increases muscle perfusion, respiratory muscle strength, and muscle fibre changes and improves functional capacity and quality of life. ${ }^{10}$ These benefits can be obtained in an even shorter study period (4 weeks), ${ }^{12}$ but the ESC/ERS guidelines of 2015 only recommended exercise therapy (ET) in class $2 \mathrm{~A}$ with a level of evidence $B$, because of the lack of a clear theory to explain how ET contributes to the improvement of symptoms and functional capacity and its effect on patient outcomes.9

Aging naturally declines aerobic capacity, but the available data suggest that older individuals have the same relative improvement in maximal aerobic capacity to aerobic training regimens. These effects are either caused by an increase in cardiac output or an increase in the extraction of oxygen at the tissue level.

Inspiratory muscle training has become an important part of pulmonary rehabilitation programmes in which various techniques are used to improve ventilation, strengthen the respiratory muscles, mobilise the thorax, and enhance secretolytic function. ${ }^{13}$ In chronic conditions such as chronic obstructive pulmonary disease and chronic heart failure, inspiratory muscle training can increase respiratory muscle strength and endurance, improve functional capacity and quality of life, and reduce dyspnoea perception. ${ }^{14}$

In patients with pulmonary hypertension who usually experience impairment of the respiratory muscles to generate force, reduced ventilatory capacity, and decreased peak oxygen uptake, anaerobic threshold, and ventilatory efficiency that cause dyspnoea and early fatigue and decrease functional capacity and quality of life, ${ }^{11}$ inspiratory muscle training can increase respiratory muscle force, which was also demonstrated in a study of patients with heart failure. Studies on how inspiratory muscle training improves functional capacity are many but the mechanisms on how it reduces PAP are very limited, except the possibility that there is an increase in pulmonary blood flow, which improves pulmonary perfusion. ${ }^{15}$ Improved pulmonary perfusion probably causes vasodilation of the pulmonary artery as can be seen in systemic artery dilation ${ }^{16}$ and reverses the mechanism of pulmonary vasoconstriction, which frequently contributes to pulmonary hypertension. ${ }^{17,18}$

In the present study, the reduction in the systolic PAP in the IMT group was also followed by a higher reduction in the maximum tricuspid regurgitant velocity (TRV) than in the CG patients, so the maximum TRV in the IMT group was lower than in the CG patients. The right ventricle function that was reflected by TAPSE also improved significantly in the IMT than in the CG patients, which was probably caused by a higher reduction in the pulmonary artery pressure and RV load. This result should be considered as an additional benefit because RV dysfunction is one factor associated with survival after valve surgery. ${ }^{19}$ Right ventricle dysfunction can persist for 6 months after mitral valve intervention because of the negative effects of $\mathrm{PH}$ on RV function. ${ }^{20}$ 
The improvement in lung function measured by the maximum inspiratory volume demonstrated a better increase in the IMT group than in the CG patients.

Regarding the safety concerns in this study, all of the procedures were supervised by trained personnel, and no adverse events occurred. Based on the results of this study, respiratory training with incentive spirometry could be added to standard exercise training programmes to achieve faster and better reduction in $\mathrm{PH}$ and improvements in functional capacity.

There were some limitations to this study. First, the sample size was relatively small. Second, there were no data regarding pulmonary embolisms that could affect PAP and right ventricle function and other potential confounders of the study results. Third, the measurement of functional capacity used a simple sub-maximum exercise testing (6-MWT) rather than office-based test which directly and accurately measure VO2max (cardiopulmonary exercise test). Forth, complete pulmonary functional testing was not conducted, except MIV which could be obtained with the equipment used in this study.

\section{Conclusion}

Adding inspiratory muscle training to a usual exercise programme resulted in faster recovery from residual pulmonary hypertension and higher increase in functional capacity.

\section{Acknowledgement}

Special gratitude for all staffs in the Cardiovascular Prevention and Rehabilitation Unit.

\section{Conflict of Interest}

None.

\section{Funding}

This paper received no specific grant from any funding agency, commercial or not-for-profit sectors.

\section{References}

1. Fawzy ME, Hassan W, Stefadouros M, Moursi M, El Shaer F, Chaudhary MA. Prevalence and fate of severe pulmonary hypertension in 559 consecutive patients with severe rheumatic mitral stenosis undergoing mitral balloon valvotomy. J Heart Valve Dis. 2004;13:942-7.

2. Dzudie A, Kengne AP, Thienemann F, Sliwa K. Predictors of hospitalisations for heart failure and mortality in patients with pulmonary hypertension associated with left heart disease: a systematic review. BMJ Open. 2014;4:e004843.

3. Kainuma S, Taniguchi K, Toda K, Funatsu $\mathrm{T}$, Kondoh $\mathrm{H}$, Nishino $\mathrm{M}$, et al. Pulmonary hypertension predicts adverse cardiac events after restrictive mitral annuloplasty for severe functional mitral regurgitation. J Thorac Cardiovasc Surg. 2011;142:783-92.

4. Guazzi M, Galie N. Pulmonary hypertension in left heart disease. Eur Respir Rev. 2012;21:338-46.

5. Townsley MI, Fu Z, Mathieu-Costello O, West JB. Pulmonary microvascular permeability. Responses to high vascular pressure after induction of pacing-induced heart failure in dogs. Circ Res. 1995;77:317-25.

6. Saxena P, Luthra S, Dhaliwal RS, Rana SS, Behera D. Early changes in pulmonary functions after mitral valve replacement. Ann Thorac Med. 2007;2:111-7.

7. Mereles D, Ehlken N, Kreuscher S, Ghofrani $S$, Hoeper MM, Halank M, et al. Exercise and respiratory training improve exercise capacity and quality of life in patients with severe chronic pulmonary hypertension. Circulation. 2006;114:1482-9.

8. Grunig E, Lichtblau M, Ehlken N, Ghofrani HA, Reichenberger F, Staehler G, et al. Safety and efficacy of exercise training in various forms of pulmonary hypertension. Eur Respir J. 2012;40:84-92.

9. Galie N, Humbert M, Vachiery JL, Gibbs S, Lang I, Torbicki A, et al. 2015 ESC/ERS Guidelines for the diagnosis and treatment of pulmonary hypertension: The Joint Task Force for the Diagnosis and Treatment of Pulmonary Hypertension of the European Society of Cardiology (ESC) and the European Respiratory Society (ERS): Endorsed by: Association for European Paediatric and Congenital 
Cardiology (AEPC), International Society for Heart and Lung Transplantation (ISHLT). Eur Respir J. 2015;46:903-75.

10. Babu AS, Padmakumar R, Maiya AG, Mohapatra AK, Kamath RL. Effects of Exercise Training on Exercise Capacity in Pulmonary Arterial Hypertension: A Systematic Review of Clinical Trials. Heart Lung Circ. 2016;25:333-41.

11. Bauer R, Dehnert C, Schoene P, Filusch A, Bartsch P, Borst MM, et al. Skeletal muscle dysfunction in patients with idiopathic pulmonary arterial hypertension. Respir Med. 2007;101:2366-9.

12. Bussotti M, Gremigni P, Pedretti RFE, Kransinska P, Di Marco S, Corbo P, et al. Effects of an Outpatient Service Rehabilitation Programme in Patients Affected by Pulmonary Arterial Hypertension: An Observational Study. Cardiovasc Hematol Disord Drug Targets. 2017;17:3-10.

13. Ries AL, BauldoffGS, Carlin BW, Casaburi R, Emery CF, Mahler DA, et al. Pulmonary Rehabilitation: Joint ACCP/AACVPR Evidence-Based Clinical Practice Guidelines. Chest. 2007;131:4S-42S.

14. Bosnak-Guclu M, Arikan H, Savci S, Inal-Ince D, Tulumen E, Aytemir K, et al. Effects of inspiratory muscle training in patients with heart failure. Respir Med. 2011;105:1671-81.

15. Ley S, Fink C, Risse F, Ehlken N, Fischer C, LeyZaporozhan J, et al. Magnetic resonance imaging to assess the effect of exercise training on pulmonary perfusion and blood flow in patients with pulmonary hypertension. Eur Radiol. 2013;23:324-31.

16. Ferreira JB, Plentz RD, Stein C, Casali KR, Arena R, Lago PD. Inspiratory muscle training reduces blood pressure and sympathetic activity in hypertensive patients: a randomized controlled trial. Int J Cardiol. 2013;166:61-7.

17. Ward JP, McMurtry IF. Mechanisms of hypoxic pulmonary vasoconstriction and their roles in pulmonary hypertension: new findings for an old problem. Curr Opin Pharmacol. 2009;9:287-96.

18. Hussain A, Suleiman MS, George SJ, Loubani M, Morice A. Hypoxic Pulmonary Vasoconstriction in Humans: Tale or Myth. Open Cardiovasc Med J. 2017;11:1-13.

19. Kammerlander AA, Marzluf BA, Graf A, Bachmann A, Kocher A, Bonderman D, et al. Right ventricular dysfunction, but not tricuspid regurgitation, is associated with outcome late after left heart valve procedure. J Am Coll Cardiol. 2014;64:2633-42.

20. Hyllen S, Nozohoor S, Ingvarsson A, Meurling C, Wierup P, Sjogren J. Right ventricular performance after valve repair for chronic degenerative mitral regurgitation. Ann Thorac Surg. 2014;98:2023-30. 